REVISTA DE DERECHO UNED, NÚM. 24, 2019

\title{
LA REGULACIÓN DE LOS NUEVOS ALIMENTOS Y NANOMATERIALES DESDE LA PERSPECTIVA DE LA PROTECCIÓN DE LA SALUD
}

THE REGULATION OF NOVEL FOOD AND NANOMATERIALS FROM THE POINT OF VIEW OF THE PROTECTION OF HEALTH

\author{
Amalia Balaguer Pérez ${ }^{1}$ \\ Investigadora en UNED
}

\begin{abstract}
Resumen: La circulación e introducción de alimentos provenientes de otros países, la utilización de nuevos ingredientes y las tecnologías para modificar los alimentos son aspectos que pueden tener un impacto en la salud de las personas. En el ámbito de los nuevos alimentos, el Reglamento, de 1997, necesitaba una actualización. La actualización del Reglamento significa, por tanto, una mayor protección de la salud, ya que se han introducido categorías de alimentos que anteriormente no estaban reguladas, como los alimentos consistentes en nanomateriales artificiales. Sin embargo, deben considerarse algunas cuestiones problemáticas en relación con este Reglamento. El presente artículo analiza los cambios del nuevo Reglamento de nuevos alimentos desde el punto de vista de la protección de la salud, teniendo en cuenta los principios generales de la regulación alimentaria y centrándose en los nanomateriales artificiales como nuevos alimentos, así como en las dificultades que hay en torno a su definición.
\end{abstract}

Palabras clave: nuevos alimentos, nanomateriales, Unión Europea, alimentos

1 A. B. P. agradece el apoyo del Programa «Ramón y Cajal» (RYC) 2015 (RYC-201518821).

(C) UNED. Revista de Derecho UNED, núm. 24, 2019 
Abstract: The movement and introduction of food from other countries, the use of new ingredients and the use of technologies to modify food are aspects that can have an impact on people's health. In the field of novel food, the Regulation, from 1997, needed an update. The update of the Regulation means, therefore, a greater protection of health, since food categories that were not regulated before have been introduced, like food consisting of engineered nanomaterials. However, we must consider some problematic issues related to this Regulation. This article analyses the changes in the new Regulation on novel food from the point of view of the protection of health, taking into account the general principles of food law and focusing on engineered nanomaterials as novel food, as well as on the difficulties regarding its definition.

Keywords: novel food, nanomaterials, European Union, food

Recepción original: 01/02/2019

Aceptación original: 22/04/2019

Sumario: I. Introducción. II. La regulación de la Unión Europea desde el punto de vista de la protección de la salud. III. Los principios generales de la regulación alimentaria y su relación con los nanomateriales. IV. Reglamento (UE) 2015/2283 relativo a los nuevos alimentos. IV. A. Definición de nanomaterial. IV. B. Los nuevos alimentos. IV. C. Procedimiento. V. Conclusiones

\section{INTRODUCCIÓN}

Los avances tecnológicos, la circulación e introducción de alimentos provenientes de otros países y la utilización de nuevos ingredientes, entre otros, son aspectos directamente relacionados con la alimentación y que pueden tener un impacto en la salud de las personas.

En un contexto en el que los alimentos son proporcionados de una manera cada vez más global, donde la exportación e importación de alimentos ocurre de manera continua, y donde se introducen tecnologías para modificar el sabor, las condiciones o la conservación de los alimentos ${ }^{2}$, la regulación alimentaria es fundamental

2 De acuerdo con CORDIS, Ventajas y desventajas de los nanoalimentos: «La nanotecnología puede emplearse para mejorar el sabor y la textura de los alimentos, reducir su contenido en grasas o encapsular ciertos nutrientes como vitaminas para impedir que se degraden durante la vida útil del producto. Asimismo, se pueden emplear nanomateriales para fabricar envases que conserven mejor y por más 
para garantizar que la población adquiera alimentos seguros. Más específicamente, algunos de estos aspectos se relacionan con el campo de los nuevos alimentos. En este ámbito, «las diferencias entre los Derechos nacionales en relación con la evaluación de la seguridad y la autorización de nuevos alimentos pueden obstaculizar la libre circulación de esos alimentos y dar lugar a inseguridad jurídica y a una competencia desleal $»^{3}$, por lo que es necesaria una regulación a nivel europeo que establezca un marco coherente para la introducción de nuevos alimentos en los diferentes Estados miembros, ya que, como se afirma en el Reglamento 178/2002 por el que se establecen los principios y los requisitos generales de la legislación alimentaria, «sólo puede conseguirse la libre circulación de alimentos y piensos dentro de la Comunidad si los requisitos de seguridad alimentaria y de los piensos no difieren significativamente de un Estado miembro a otro» ${ }^{4}$.

En el ámbito de los nuevos alimentos, el Reglamento, de 1997, necesitaba una actualización. En el Reglamento de 2015 se han introducido categorías de nuevos alimentos que no estaban reguladas anteriormente; es el caso de los nanomateriales, que se usan en la alimentación para modificar los alimentos ${ }^{5}$, pero cuyos efectos no son del todo conocidos. Era, por tanto, necesario regular este campo, ya que «los productos de nanotecnología podrían tener

tiempo la frescura del producto. Es más, pueden crearse envases inteligentes dotados de nanosensores que informen al consumidor del estado en que se encuentra el producto del interior». Disponible en: https://cordis.europa.eu/news/rcn/30485_ es.html

${ }^{3}$ Reglamento (UE) 2015/2283 del Parlamento Europeo y del Consejo, de 25 de noviembre de 2015, relativo a los nuevos alimentos, por el que se modifica el Reglamento (UE) n. ${ }^{\circ} 1169 / 2011$ del Parlamento Europeo y del Consejo y se derogan el Reglamento (CE) n. ${ }^{\circ}$ 258/97 del Parlamento Europeo y del Consejo y el Reglamento (CE) n. ${ }^{\circ}$ 1852/2001 de la Comisión, (1).

${ }^{4}$ Reglamento (CE) n. ${ }^{\circ}$ 178/2002 del Parlamento Europeo y del Consejo, de 28 de enero de 2002, por el que se establecen los principios y los requisitos generales de la legislación alimentaria, se crea la Autoridad Europea de Seguridad Alimentaria y se fijan procedimientos relativos a la seguridad alimentaria, (3).

5 Así, se citan como desarrollos en el campo de la nanotecnología en relación con los alimentos aquellos que «hasta ahora han estado dirigidos a alterar la textura de los componentes alimentarios, encapsular los componentes o aditivos alimentarios, desarrollar nuevos sabores y sensaciones, controlar la liberación de sabores y/o incrementar la biodisponibilidad de los componentes nutricionales», CHAUDHRY, Q., SCOTTER, M., BLACKBURN, J., ROSS, B., BOXALL, A., CASTLE, L., AITKEN, R., WATKINS, R., "Applications and implications of nanotechnologies for the food sector», Food Additives and Contaminants, 25:3, 2008, págs. 241-258, pág. 243 (traducción propia): «The main developments so far been aimed at altering the texture of food components, encapsulating food components or additives, developing new tastes and sensations, controlling the release of flavours, and/or increasing the bioavailability of nutritional components». 
un impacto sustancial en el sector de los alimentos y piensos, ofreciendo beneficios potenciales para la industria y el consumidor, aunque deben considerarse los posibles riesgos (...) Las características y propiedades específicas de los nanomateriales necesitan considerarse para cualquier riesgo de salud potencial ${ }^{6}$. Se ha afirmado que "aunque todavía se está recopilando y evaluando información, algunos resultados sugieren que la exposición dietética a los NMAs [nanomateriales artificiales] puede tener resultados adversos para la salud ${ }^{7}$. Por tanto, existe incertidumbre en este campo, aunque parece entenderse que puede haber riesgos potenciales asociados.

Desde el Reglamento 2015/2283 los alimentos que consisten en nanomateriales se consideran nuevos alimentos. La actualización del Reglamento, por tanto, significa una mayor protección de la salud, ya que se han introducido categorías de alimentos, como aquellos consistentes en nanomateriales, que anteriormente no estaban regulados y que, en consecuencia, no necesitaban pasar el proceso de autorización para nuevos alimentos.

El Reglamento incluye una definición de nanomaterial artificial que, como se verá más adelante, no es igual a la definición de nanomaterial que aparece en otros ámbitos. Además de introducirse nuevas categorías de alimentos, se ha cambiado el procedimiento de autorización, de manera que el procedimiento actual es centralizado. El nuevo procedimiento se extiende menos en el tiempo, pero, no obstante, se afirma que todavía se espera que lleve al menos dos años ${ }^{8}$.

${ }^{6}$ EFSA, Nanotechnology: «Nanotechnology products could have a substantial impact on the food and feed sector in the future, potentially offering benefits for industry and the consumer, although possible risks need to be considered. (...) The specific properties and characteristics of nanomaterials need to be considered for any potential health risks». Información disponible en: https:/www.efsa.europa.eu/ en/topics/topic/nanotechnology

También: CORDIS, Ventajas y desventajas de los nanoalimentos: «La nanotecnología tiene la capacidad de mejorar los alimentos que consumimos haciéndolos más sabrosos, sanos y nutritivos. Sin embargo, no se sabe mucho acerca del modo en que las nanopartículas se comportan en el organismo, ni tampoco sobre los efectos tóxicos que podrían tener (...) Por otra parte, la adición de nanomateriales a los alimentos tampoco está exenta de riesgos». Disponible en: https://cordis.europa.eu/ news/rcn/30485_es.html

${ }^{7}$ GRIEGER, K.D., HANSEN, S.F., MORTENSEN, N.P., CATES, S., KOWALCYK, B., "International Implications of Labeling Foods Containing Engineered Nanomaterials», Journal of Food Protection, Vol. 79, No. 5, 2016, págs. 380-842, pág. 833: "Although data are still being gathered and evaluated, some results suggest that dietary exposure to ENMs may result in adverse health outcomes».

8 BOER, A., BAST, A., «Demanding safe foods - Safety testing under the novel food regulation (2015/2283)», Trends in Food Science \& Technology 72, 2018, 
El presente artículo analiza estos cambios del Reglamento de nuevos alimentos desde el punto de vista de la salud, teniendo en cuenta los principios generales de la regulación alimentaria y centrándose en los nanomateriales como parte de los nuevos alimentos, así como en las dificultades que hay en torno a su definición.

\section{LA REGULACIÓN DE LA UNIÓN EUROPEA DESDE EL PUNTO DE VISTA DE LA PROTECCIÓN DE LA SALUD}

En relación con la salud, la Unión Europea tiene ciertas obligaciones y competencias respecto a la protección de la salud. La «protección y mejora de la salud humana» es una competencia de apoyo de acuerdo con el artículo 6 del TFUE que indica que «la Unión dispondrá de competencia para llevar a cabo acciones con el fin de apoyar, coordinar o complementar la acción de los Estados miembros» en este ámbito. Las competencias compartidas entre la Unión y los Estados miembros (art. 4 TFUE) en los ámbitos del mercado interior y la protección de los consumidores y en los asuntos comunes de seguridad en materia de salud pública implican también la regulación de la Unión en el campo de la alimentación. En este sentido, el artículo 169.1 del TFUE indica que "para promover los intereses de los consumidores y garantizarles un alto nivel de protección, la Unión contribuirá a proteger la salud, la seguridad y los intereses económicos de los consumidores, así como a promover su derecho a la información, a la educación y a organizarse para salvaguardar sus intereses», ya que la salud está relacionada con la protección de los consumidores, ámbito cercano a la regulación alimentaria. Las personas, en su condición de consumidoras de alimentos, necesitan que se proteja su salud en relación a estos y se evite la comercialización de alimentos no seguros.

En este sentido, el artículo 168.1 del TFUE establece que «al definirse y ejecutarse todas las políticas y acciones de la Unión se garantizará un alto nivel de protección de la salud humana» ${ }^{9}$ y el ar-

págs. 125-133, pág. 127: «the new and centralised authorisation procedure consists of fewer steps, which should speed up the decision-making process upon the authorisation of a NF and make it less time- and resource consuming for both industrial and decision makers» and "although the new process contains fewer steps, the authorisation procedure is still expected to take at least two years».

9 También en el artículo 114 se indica que «1. Salvo que los Tratados dispongan otra cosa, se aplicarán las disposiciones siguientes para la consecución de los objetivos enunciados en el artículo 26. El Parlamento Europeo y el Consejo, con arreglo al procedimiento legislativo ordinario y previa consulta al Comité Económico y Social, adoptarán las medidas relativas a la aproximación de las disposiciones legales,

(C) UNED. Revista de Derecho UNED, núm. 24, 2019 
tículo 9 del TFUE que «en la definición y ejecución de sus políticas y acciones, la Unión tendrá en cuenta las exigencias relacionadas con la promoción de un nivel de empleo elevado, con la garantía de una protección social adecuada, con la lucha contra la exclusión social y con un nivel elevado de educación, formación y protección de la salud humana».

La Unión Europea tiene, por tanto, la obligación de garantizar un alto nivel de protección de la salud humana en sus políticas y acciones. Tiene además la obligación de contribuir a la protección de la salud de las personas consumidoras.

La protección de la salud es además un derecho, de acuerdo con el artículo 35 de la Carta de Derechos Fundamentales de la Unión Europea:

\section{Protección de la salud}

Toda persona tiene derecho a acceder a la prevención sanitaria y a beneficiarse de la atención sanitaria en las condiciones establecidas por las legislaciones y prácticas nacionales. Al definirse y ejecutarse todas las políticas y acciones de la Unión se garantizará un nivel elevado de protección de la salud humana.

La relación entre salud y alimentación requiere que se establezcan unas normas en materia de seguridad alimentaria. En este sentido, en el contexto del mercado interior «no podría haber libre comercio si cada producto tuviese que controlarse en cada país de acuerdo con normas diferentes» ${ }^{10}$.

En el ámbito de los nuevos alimentos, «las diferencias entre los Derechos nacionales en relación con la evaluación de la seguridad y la autorización de nuevos alimentos pueden obstaculizar la libre circulación de esos alimentos y dar lugar a inseguridad jurídica y a una

reglamentarias y administrativas de los Estados miembros que tengan por objeto el establecimiento y el funcionamiento del mercado interior (...) 3. La Comisión, en sus propuestas previstas en el apartado 1 referentes a la aproximación de las legislaciones en materia de salud, seguridad, protección del medio ambiente y protección de los consumidores, se basará en un nivel de protección elevado, teniendo en cuenta especialmente cualquier novedad basada en hechos científicos. En el marco de sus respectivas competencias, el Parlamento Europeo y el Consejo procurarán también alcanzar ese objetivo».

10 European Commission, "The European Union explained. Food safety. From farm to fork: safe and healthy food for everyone. Ensuring a high level of protection of human health and consumers interests», 2014, pág. 3: "There could not be any free trade if every product had to be controlled in each country according to different rules». 
competencia desleal» ${ }^{11}$. En relación a los nanomateriales artificiales, se establece en el Reglamento 2015/2283, debido a este necesidad de coherencia, una definición, como se verá más adelante.

\section{LOS PRINCIPIOS GENERALES DE LA REGULACIÓN ALIMENTARIA Y SU RELACIÓN CON LOS NANOMATERIALES}

Los principios generales de la regulación alimentaria en la Unión Europa se encuentran en el Reglamento 178/2002 del Parlamento Europeo y del Consejo, de 28 de enero de 2002, por el que se establecen los principios y los requisitos generales de la legislación alimentaria, se crea la Autoridad Europea de Seguridad Alimentaria y se fijan procedimientos relativos a la seguridad alimentaria. De acuerdo con el artículo 1.1. del Reglamento, este «proporciona la base para asegurar un nivel elevado de protección de la salud de las personas y de los intereses de los consumidores en relación con los alimentos, teniendo en cuenta, en particular, la diversidad del suministro de alimentos, incluidos los productos tradicionales, al tiempo que se garantiza el funcionamiento eficaz del mercado interior». Su objetivo principal, por tanto, es salvaguardar la protección de la salud de las personas, lo que se especifica en el artículo 5, que recoge los objetivos generales de la legislación alimentaria: «la legislación alimentaria perseguirá uno o varios de los objetivos generales de lograr un nivel elevado de protección de la vida y la salud de las personas, así como de proteger los intereses de los consumidores» (5.1.)

Dos principios son fundamentales respecto a la seguridad alimentaria, según se establece en este reglamento.

Uno de ellos es el análisis del riesgo (art. $6^{12}$ ), que implica que la legislación alimentaria se basará en este, salvo excepciones, y que se

${ }^{11}$ Reglamento (UE) 2015/2283 del Parlamento Europeo y del Consejo de 25 de noviembre de 2015, considerandos, 1.

12 1. Con el fin de lograr el objetivo general de un nivel elevado de protección de la salud y la vida de las personas, la legislación alimentaria se basará en el análisis del riesgo, salvo que esto no convenga a las circunstancias o la naturaleza de la medida legislativa.

2. La determinación del riesgo se basará en las pruebas científicas disponibles y se efectuará de una manera independiente, objetiva y transparente.

3. Con objeto de alcanzar los objetivos generales de la legislación alimentaria establecidos en el artículo 5, la gestión del riesgo tendrá en cuenta los resultados de la determinación del riesgo y, en particular, los dictámenes de la Autoridad Europea de Seguridad Alimentaria mencionada en el artículo 22, el principio de cautela cuando sean pertinentes las condiciones mencionadas en el apartado 1 del artículo 7 , así como otros factores relevantes para el tema de que se trate.

(C) UNED. Revista de Derecho UNED, núm. 24, 2019 
determinará el riesgo mediante pruebas científicas. En relación con los nuevos alimentos, este principio se manifiesta en la condición por la que sólo se autorizará un nuevo alimento si este, "sobre la base de las pruebas científicas disponibles (...) no plantea un riesgo para la salud de las personas» ${ }^{13}$.

Por otra parte, otro de los principios generales es el principio de cautela, por el que si se observa, tras evaluar la información disponible, que existe la posibilidad de que haya efectos nocivos para la salud, pero hay incertidumbre científica, se podrán adoptar medidas provisionales de gestión del riesgo hasta que esté disponible información científica adicional que permita determinar el riesgo de manera más exhaustiva.

El principio de cautela o precaución estaba en principio relacionado con el medio ambiente ${ }^{14}$, pero se ha extendido a otros ámbitos: "pretende garantizar un elevado nivel de protección del medio ambiente mediante tomas de decisión preventivas en caso de riesgo. No obstante, en la práctica, su ámbito de aplicación es mucho más amplio y se extiende asimismo a la política de los consumidores, a la legislación de la Unión Europea (UE) relativa a los alimentos, a la salud humana, animal y vegetal» ${ }^{15}$.

Para entender el alcance de este principio hay que acudir a la Comunicación de la Comisión sobre el recurso al principio de precaución, puesto que el Tratado sólo lo menciona. Este principio no limita su ámbito a los riesgos a corto o medio plazo sino que «se refiere también a cuestiones a largo plazo e incluso ligadas al bienestar de las generaciones futuras» ${ }^{16}$.

Respecto a la salud, debe tenerse también en cuenta que los efectos de ciertos alimentos o materiales en la salud pueden producirse a largo plazo y que «a la hora de determinar si un alimento es nocivo para la salud, se tendrán en cuenta los probables efectos inmediatos y a corto y largo plazo de ese alimento» ${ }^{17}$.

13 Reglamento 2015/2283, art. 7.a.

${ }^{14}$ Aparece así en el TFUE Art. 191.2: «La política de la Unión en el ámbito del medio ambiente tendrá como objetivo alcanzar un nivel de protección elevado, teniendo presente la diversidad de situaciones existentes en las distintas regiones de la Unión. Se basará en los principios de cautela y de acción preventiva, en el principio de corrección de los atentados al medio ambiente, preferentemente en la fuente misma, y en el principio de quien contamina paga.»

15 Principio de precaución, summaries of EU legislation. Disponible en: https:// eur-lex.europa.eu/legal-content/ES/TXT/?uri=LEGISSUM\%3Al32042

${ }^{16}$ Comunicación de la Comisión sobre el recurso al principio de precaución /* $\mathrm{COM} / 2000 / 0001$ final $* /,(1)$.

17 Reglamento 178/2002, art. 14.4.a. 
El principio de cautela está especialmente relacionado con los nanomateriales ya que, como se ha afirmado en la introducción de este artículo, sus efectos no son del todo conocidos pero puede haber riesgos potenciales en relación a estos.

\section{REGLAMENTO (UE) 2015/2283 RELATIVO A LOS NUEVOS ALIMENTOS}

Este Reglamento tiene como objeto regular «la comercialización de nuevos alimentos en la Unión» (art. 1.1) y su finalidad es "garantizar el buen funcionamiento del mercado interior a la vez que se proporciona un elevado nivel de protección de la salud y de los intereses de los consumidores» (art. 1.2).

El objetivo principal es garantizar el buen funcionamiento del mercado interior, pero la protección de la salud es un objetivo que está relacionado con este ya que la circulación de alimentos y la introducción de nuevos alimentos pueden influir en la salud de las personas.

Aunque el Reglamento es del año 2015, la mayoría de sus disposiciones son aplicables a partir de enero de 2018 y sustituye al Reglamento (CE) n. ${ }^{\circ}$ 258/97 del Parlamento Europeo y del Consejo de 27 de enero de 1997 sobre nuevos alimentos y nuevos ingredientes alimentarios. Las nuevas tecnologías tienen ahora un papel más relevante en relación con los alimentos que en la fecha del anterior Reglamento. Debe tenerse en cuenta que en este no se mencionaban los nanomateriales.

Entre las novedades que introduce este nuevo reglamento se encuentran, por tanto, aquellas referidas a los nanomateriales. Era necesaria una actualización ya que el campo de los nuevos alimentos ha cambiado en los últimos años, entre otras cosas en lo referente a los nanomateriales, si bien también se han añadido otras nuevas categorías de nuevos alimentos. Los alimentos que consistan en nanomateriales artificiales se consideran nuevos alimentos de acuerdo con este Reglamento (art. 3.2.viii), así como las vitaminas, minerales y otras sustancias utilizadas con arreglo a la Directiva 2002/46/ CE, al Reglamento (CE) no 1925/2006 o al Reglamento (UE) no $609 / 2013$ que contengan o consistan en nanomateriales artificiales (art. 3.2.ix). A este respecto, hay una definición de nanomaterial artificial a efectos de este Reglamento. 


\section{IV.A. Definición de nanomaterial}

En cuanto a la definición de nanomaterial artificial, aunque el Reglamento lo define para el ámbito de los nuevos alimentos, hay que tener en cuenta que no hay una definición única de nanomaterial que se aplique de manera general a nivel europeo.

La definición de nanomaterial artificial que encontramos en este Reglamento es «cualquier material producido intencionadamente que tenga una o más dimensiones del orden de los $100 \mathrm{~nm}$ o menos o que esté compuesto de partes funcionales diferenciadas, internamente o en superficie, muchas de las cuales tengan una o más dimensiones del orden de $100 \mathrm{~nm}$ o menos, incluidas estructuras, aglomerados o agregados, que pueden tener un tamaño superior a los $100 \mathrm{~nm}$, pero conservan propiedades que son características de la nanoescala» ${ }^{18}$.

Esta definición es diferente a la definición de nanomaterial que se encuentra en otros ámbitos, pero, no obstante, el Reglamento (UE) N. ${ }^{\circ} 1169 / 2011$ del Parlamento Europeo y del Consejo de 25 de Octubre, ha sido modificado por el Reglamento 2015/2283, que ha incluido una remisión a la definición de nanomaterial artificial que aparece en el propio Reglamento de 2015, indicando que será de aplicación «la definición de "nanomateriales artificiales» tal como se formula en el artículo 3, apartado 2, letra f)» ${ }^{19}$. El Reglamento de 2015 destaca la necesidad de coherencia respecto a la definición de nanomaterial, en este ámbito: «El término «nanomaterial artificial» está definido actualmente en el Reglamento (UE) no 1169/2011 del Parlamento Europeo y del Consejo. Por razones de coherencia, es importante garantizar que haya solamente una definición de nanomaterial artificial en el ámbito de la legislación sobre alimentos. El marco legislativo adecuado para incluir esa definición es el presente Reglamento. En consecuencia, la definición de nanomaterial artificial y la correspondiente delegación de poderes en la Comisión, deben suprimirse del Reglamento (UE) no 1169/2011 y sustituirse por una remisión a la definición del presente Reglamento» (10).

18 Reglamento 2015/2283, art. 3.2.f, que continúa diciendo: «Entre las propiedades características de la nanoescala figuran:

i) las relacionadas con la gran superficie específica de los materiales considerados, $y / o$

ii) las propiedades físico-químicas específicas que son distintas de la forma no nanotecnológica del mismo material».

19 Art. 2. 1. h. 
No obstante, nos encontramos, también dentro del ámbito alimentario, cierta ausencia de definiciones respecto a esta temática, pues el Reglamento (UE) No 10/2011 de la Comisión, de 14 de enero de 2011, sobre materiales y objetos plásticos destinados a entrar en contacto con alimentos, menciona las «sustancias en nanoforma» pero no las define (art. 9.2, art. 13.4.b, art. 14.3.b) y el Reglamento (CE) No 450/2009 de la Comisión, de 29 de mayo de 2009, sobre materiales y objetos activos e inteligentes destinados a entrar en contacto con alimentos menciona las nanopartículas pero tampoco las define (considerando 14).

Otro aspecto a considerar es que la definición que se encuentra en el Reglamento sobre nuevos alimentos está sujeta a cambios, dependiendo del progreso técnico: «a fin de alcanzar los objetivos del presente Reglamento, la Comisión, mediante actos delegados adoptados con arreglo al artículo 32, ajustará y adaptará la definición de nanomateriales artificiales a que se refiere el artículo 3, apartado 2 , letra $\mathrm{f}$ ), a los progresos técnicos y científicos o a las definiciones acordadas a escala internacional» (art. 31). Se trata de una medida necesaria al tratarse de un campo relativamente nuevo. Desde el punto de vista de la protección de la salud, por tanto, esta definición debe estar abierta a actualización, pues de la definición depende que un material se considere nanomaterial o no y, por tanto, entre dentro del ámbito de este Reglamento. Sin embargo, la posibilidad de que la definición pueda necesitar adaptarse implica también una mayor inseguridad, pues materiales que no se consideren actualmente nanomateriales pueden serlo en el futuro.

Por otra parte, de manera más genérica y no limitada al ámbito alimentario, la Recomendación de la Comisión, de 18 de octubre de 2011, (2011/696/UE), relativa a la definición de nanomaterial, define nanomaterial de la siguiente manera:

«Por «nanomaterial» se entiende un material natural, secundario o fabricado que contenga partículas, sueltas o formando un agregado o aglomerado y en el que el $50 \%$ o más de las partículas en la granulometría numérica presente una o más dimensiones externas en el intervalo de tamaños comprendido entre $1 \mathrm{~nm}$ y $100 \mathrm{~nm}$.

En casos específicos y cuando se justifique por preocupaciones de medio ambiente, salud, seguridad o competitividad, el umbral de la granulometría numérica del $50 \%$ puede sustituirse por un umbral comprendido entre el $1 \%$ y el $50 \% » .^{20}$

${ }^{20}$ (2). El punto 3 prosigue: «3. No obstante lo dispuesto en el punto 2, los fullerenos, los copos de grafeno y los nanotubos de carbono de pared simple con una o más dimensiones externas inferiores a $1 \mathrm{~nm}$ deben considerarse nanomateriales». 
Se trata de una definición genérica de nanomaterial, mientras que en el Reglamento del 2015 se define únicamente «nanomaterial artificial». Esta definición del Reglamento 2015/2283 debe ponerse en relación con el ámbito de aplicación de este. Se establece un ámbito concreto en el que se aplica la definición de nanomaterial artificial. Específicamente, se excluye del ámbito de aplicación del Reglamento relativo a los nuevos alimentos, a «los alimentos cuando y en la medida en que se usen como: i) enzimas alimentarias incluidas en el ámbito de aplicación del Reglamento (CE) n. ${ }^{\circ}$ 1332/2008, ii) aditivos alimentarios incluidos en el ámbito de aplicación del Reglamento (CE) n. ${ }^{\circ} 1333 / 2008 »$, entre otros ${ }^{21}$.

Respecto a los aditivos y la definición de nanomaterial, hay que mencionar que el Reglamento Delegado (UE) n. ${ }^{\circ}$ 1363/2013 de la Comisión, de 12 de diciembre de 2013, por el que se modifica el Reglamento (UE) n. ${ }^{\circ}$ 1169/2011 del Parlamento Europeo y del Consejo, sobre la información alimentaria facilitada al consumidor, entendía que no debían incluirse ciertos aditivos alimentarios en la definición de nanomaterial artificial, indicando que «los aditivos alimentarios incluidos en las listas de la Unión en virtud de los Reglamentos (UE) no $1129 / 2011$ y (UE) no 1130/2011 no deben ir obligatoriamente acompañados de la palabra "nano" en la lista de ingredientes ni deben incluirse en la definición de "nanomaterial artificial" » si bien "esta excepción no debe aplicarse a los aditivos alimentarios insertados en estas listas en una fecha posterior, incluidas las nuevas entradas con arreglo al artículo 12 del Reglamento (CE) no 1333/2008» (12) .

La Resolución del Parlamento Europeo, de 12 de marzo de 2014, sobre el Reglamento Delegado de la Comisión, de 12 de diciembre de 2013, por el que se modifica el Reglamento (UE) no 1169/2011 del Parlamento Europeo y del Consejo, sobre la información alimentaria

${ }^{21}$ Art.2.2.: «El presente Reglamento no se aplica a:

a) los alimentos modificados genéticamente incluidos en el ámbito de aplicación del Reglamento (CE) no 1829/2003;

b) los alimentos cuando y en la medida en que se usen como:

i) enzimas alimentarias incluidas en el ámbito de aplicación del Reglamento (CE) no 1332/2008,

ii) aditivos alimentarios incluidos en el ámbito de aplicación del Reglamento (CE) no 1333/2008,

iii) aromas alimentarios incluidos en el ámbito de aplicación del Reglamento (CE) no 1334/2008,

iv) disolventes de extracción usados o destinados al uso en la producción de alimentos o ingredientes alimentarios e incluidos en el ámbito de aplicación de la Directiva 2009/32/CE». 
facilitada al consumidor, en lo relativo a la definición de «nanomaterial artificial» (C(2013)08887 - 2013/2997(DEA)) consideró «que, en la actualidad, son precisamente los aditivos alimentarios los que pueden estar presentes como nanomateriales en los alimentos (...) esta exención general anula las disposiciones de etiquetado para todos los aditivos alimentarios que son nanomateriales artificiales; que esto priva al acto legislativo de su principal «efecto útil» y que es contrario al objetivo fundamental de la Directiva, a saber, perseguir un alto nivel de protección de la salud y los intereses de los consumidores ofreciendo una base para que los consumidores finales puedan elegir con conocimiento de causa» (F y G). Como dice esta resolución, "un aditivo alimentario o es un nanomaterial o no lo es» (J). Aunque la publicación del Reglamento Delegado se declaró sin efecto al poco tiempo de su entrada en vigor ${ }^{22}$, esta situación ilustra las dificultades en torno a la definición de nanomaterial.

Por último, también encontramos definiciones distintas de nanomaterial en regulaciones ajenas al campo alimentario, como el Reglamento (CE) n. ${ }^{\circ}$ 1223/2009 del Parlamento Europeo y del Consejo, de 30 de noviembre de 2009, sobre los productos cosméticos, que define nanomaterial como "un material insoluble o biopersistente fabricado intencionalmente que presenta una o más dimensiones externas o una estructura interna del orden de 1 a $100 \mathrm{~nm}$ » (art. 2.1.k), así como en el Reglamento (UE) n. ${ }^{\circ}$ 528/2012 del Parlamento Europeo y del Consejo, de 22 de mayo de 2012, relativo a la comercialización y el uso de los biocidas ${ }^{23}$.

La definición de nanomaterial se encuentra en relación con la protección de la salud puesto que dependiendo de la definición se considerará si un material es un nanomaterial o no y, por tanto, si tiene que cumplir condiciones específicas destinadas a la protec-

${ }^{22}$ De acuerdo con la Corrección de errores del Reglamento Delegado (UE) no 1363/2013 de la Comisión, de 12 de diciembre de 2013, por el que se modifica el Reglamento (UE) no 1169/2011 del Parlamento Europeo y del Consejo, sobre la información alimentaria facilitada al consumidor, en lo relativo a la definición de «nanomaterial artificial» (Diario Oficial de la Unión Europea L 343 de 19 de diciembre de 2013): «La publicación del Reglamento Delegado (UE) no 1363/2013 de la Comisión se considerará nula y sin efecto».

${ }^{23}$ En este, se define nanomaterial como «una sustancia activa o no activa natural o fabricada que contenga partículas, sueltas o formando un agregado o aglomerado y en el que el $50 \%$ o más de las partículas en la granulometría numérica presente una o más dimensiones externas en el intervalo de tamaños comprendido entre $1 \mathrm{~nm}$ y $100 \mathrm{~nm}$.

Los fullerenos, los copos de grafeno y los nanotubos de carbono de pared simple con una o más dimensiones externas inferiores a $1 \mathrm{~nm}$ se considerarán nanomateriales» (art. 3.1.z). 
ción de la salud. Especialmente en el caso de los nuevos alimentos, ya que todos aquellos alimentos que consistan en nanomateriales se consideran nuevos alimentos y tienen que pasar un proceso de autorización. Como afirman AMENTA y otros «el uso de definiciones distintas permite abordar los nanomateriales según las necesidades de áreas de aplicación específicas (p. ej. aplicaciones industriales o alimentarias); sin embargo, puede también llevar a confusión e inconsistencia regulatoria ya que la misma sustancia podría considerarse como NM en una legislación pero no en otra ${ }^{24}$. Esto es especialmente relevante cuando se trata de legislación diferente que afecta a una misma materia, como es el caso de aquella relativa al ámbito alimentario.

\section{IV.B. Los nuevos alimentos}

Se considera nuevo alimento en este Reglamento «todo alimento que no haya sido utilizado en una medida importante para el consumo humano en la Unión antes del 15 de mayo de 1997, con independencia de las fechas de adhesión de los Estados miembros a la Unión» (art. 3.2.a), y que esté al menos en una de las categorías establecidas, entre las que se encuentra "alimento que consista en nanomateriales artificiales, tal como se definen en la letra f) del presente apartado» (art. 3.2.viii). También «las vitaminas, minerales y otras sustancias utilizadas con arreglo a la Directiva 2002/46/CE, al Reglamento (CE) no 1925/2006 o al Reglamento (UE) no 609/2013: (...) que contengan o consistan en nanomateriales artificiales, tal como se definen en la letra f) del presente apartado» (art. 3.2.ix), en los casos establecidos por el Reglamento ${ }^{25}$.

Este sería por tanto el ámbito de aplicación de la definición de nanomaterial artificial que se encuentra en este Reglamento. Todo aquello que se considere nanomaterial artificial según la definición,

24 AMENTA, V., ASCHBERGER, K., ARENA, M., BOUWMEESTER, H., y otros, «Regulatory Aspects of Nanotechnology in the Agri/feed/food Sector in EU and non-EU Countries», Regulatory Toxicology and Pharmacology 73, págs. 463476, 2015, pág. 466: «The use of different definitions allows addressing NMs in a way that is tailored to the needs of specific application areas (e.g. food or industrial applications); however, it can also lead to confusion and regulatory inconsistency as the same substance could be regarded as NM by one legislation but not by another».

${ }_{25}$ En concreto, aquellos «a los que se haya aplicado un proceso de producción no utilizado para la producción alimentaria en la Unión antes del 15 de mayo de 1997 contemplado en el inciso vii) de la presente letra» (art. 3.2.ix). 
pero no esté dentro de estas categorías, no es relevante en relación con los nuevos alimentos y no se considerará como tal.

Se consideran nuevos alimentos aquellos que consisten en nanomateriales artificiales, lo que parece dar a entender que deben ser alimentos enteramente compuestos por nanomateriales. Sin embargo, en relación con los ingredientes, el Reglamento establece que «no deben considerarse un nuevo alimento los alimentos elaborados exclusivamente a partir de ingredientes alimentarios que no entran en el ámbito de aplicación del presente Reglamento, en particular después de un cambio de ingredientes del alimento o de su cantidad. No obstante, las modificaciones en un ingrediente alimentario que no se haya utilizado todavía para el consumo humano en una medida importante en la Unión, sí deben entrar en el ámbito de aplicación del presente Reglamento» (17).

La inclusión de nuevos alimentos en la Unión Europea sin regulación produciría una gran inseguridad respecto a estos y sus efectos en la salud. Por ello, se establece un procedimiento de autorización específico (Capítulo III del Reglamento) y se indica que «sólo los nuevos alimentos autorizados e incluidos en la lista de la Unión podrán comercializarse en la Unión como tales o utilizarse en alimentos, de conformidad con las condiciones de utilización y los requisitos de etiquetado que en ella se especifiquen» (art. 6.2). De esta manera se pretende asegurar que los nuevos alimentos son seguros, estableciendo un procedimiento único pues en los considerandos se indica que «las diferencias entre los Derechos nacionales en relación con la evaluación de la seguridad y la autorización de nuevos alimentos pueden obstaculizar la libre circulación de esos alimentos y dar lugar a inseguridad jurídica y a una competencia desleal» (1).

La incorporación de nuevas categorías de nuevos alimentos, respecto del Reglamento anterior, supone un avance porque contribuye a la protección de la salud, ya que se trata de categorías que antes no estaban reguladas y que, por tanto, no tenían que pasar el proceso de autorización.

\section{IV.C. Procedimiento}

Otro cambio de este nuevo Reglamento, respecto al anterior, es en relación al procedimiento de autorización de nuevos alimentos. Se trata de un proceso centralizado, que, de acuerdo con BOER y BAST «consta de menos pasos, lo que debería acelerar el 
proceso de toma de decisiones sobre la autorización de un NA y consumir menos recursos y tiempo para los responsables y la industria», si bien "aunque el nuevo proceso contiene menos pasos, todavía se espera que el proceso de autorización lleve al menos dos años $»^{26}$.

La dificultad del procedimiento puede influir en la protección de la salud si conlleva la entrada de alimentos no autorizados en el mercado. Aunque un procedimiento difícil y costoso no conlleva necesariamente la entrada de alimentos no autorizados en el mercado, a este respecto, y sobre el Reglamento de 1997, HYDE, HARTLEY y MILLAR han afirmado que «desde el 2002, ha habido 215 notificaciones en la base de datos del RASFF sobre nuevos alimentos no autorizados que han aparecido en el mercado en la $\mathrm{UE} »^{27}$ y sobre las posibles razones de que no se cumpla la regulación, se cuestionan si puede ser con conocimiento y deberse a los desafíos del sistema regulatorio o si es de manera involuntaria por creer que los alimentos están aprobados o no saber que hay previstos requisitos para su aprobación ${ }^{28}$.

En términos generales, en el Reglamento 258/1997 el procedimiento se iniciaba cuando la persona solicitante presentaba una solicitud al Estado miembro en el que iba a comercializar el producto y una copia de esta solicitud a la Comisión (art. 4), solicitud que debía incluir los estudios realizados respecto al alimento y cualquier otra prueba de que el alimento cumplía con los criterios establecidos, entre otra información (art. 6.1). El Estado miembro encargaba entonces una evaluación inicial a un organismo de

${ }^{26}$ BOER, A., BAST, A., "Demanding safe foods - Safety testing under the novel food regulation (2015/2283)», Trends in Food Science \& Technology 72, 2018, págs. $125-133$, pág. 127: «the new and centralised authorisation procedure consists of fewer steps, which should speed up the decision-making process upon the authorisation of a NF and make it less time- and resource consuming for both industrial and decision makers» and "although the new process contains fewer steps, the authorisation procedure is still expected to take at least two years».

${ }^{27}$ HYDE, R., HARTLEY, S., MILLAR, K., «European novel Foods Policy at a critical juncture: drawing lessons for future Novel Food Governance through a retrospective examination of Regulation 258/97», Food and Drug Law Journal, 72 (3), págs. 472-505, pág. 486: «since 2002, 215 notifications have been placed on the RASFF database concerning unauthorized novel foods appearing on the market in the EU»

28 Ibid., pág. 487: «taking the risk and knowingly placing unapproved food products on the market because of the challenges of the regulatory system» or businesses are «inadvertently contravening the requirements for approval, placing food on the market believing it is approved or not knowing that there are approval requirements». 
evaluación de alimentos cuyo nombre tenía que notificar a la Comisión o solicitaba a la Comisión que concertase con otro Estado miembro la elaboración del informe inicial (art. 6.2). El informe resultante se remitía a la Comisión, que lo remitía a su vez a los demás Estados miembros, abriéndose entonces un plazo para presentar observaciones u objeciones por parte de la Comisión o de un Estado miembro (art. 6.4.) El informe podía también establecer si se requería una evaluación complementaria (art. 6.3.). Tras la evaluación inicial, el Estado miembro informaba al solicitante de que podía "poner en el mercado el alimento o ingrediente alimentario, en caso de no requerirse la evaluación complementaria mencionada (...) y siempre que no se haya formulado ninguna objeción» por la Comisión o un Estado miembro (art. 4.2). En el caso de que se necesitara una evaluación complementaria o se formulase una objeción de conformidad, se adoptaba una decisión de autorización, con la asistencia de «el Comité Permanente de la Cadena Alimentaria y de Sanidad Animal». El proyecto de acto de ejecución se adoptaba por la Comisión si el Comité emitía un dictamen favorable (art. 7 y art. 13.2) ${ }^{29}$.

Sin embargo, en el nuevo procedimiento, «los explotadores de empresas alimentarias» deben consultar «al Estado miembro en el que quieren comercializar primero el nuevo alimento» si no están seguros de «si un alimento que quieren comercializar en la Unión entra en el ámbito de aplicación del presente Reglamento» (art. 4). La solicitud se dirige directamente a la Comisión, que la pondrá a disposición de los Estados miembros y hará público un resumen de esta, si bien existe también la posibilidad de que el procedimiento comience a iniciativa de esta (art. 10.1). Esta solicitud, deberá contener las «pruebas científicas que demuestren que el nuevo alimento no plantea un riesgo para la salud de las personas» (art. 10.2.e), entre otra información, por lo que siguen siendo las empresas solicitantes las que deben probar que el alimento no es un riesgo. La Autoridad Europea de Seguridad Alimentaria, que no existía cuando el anterior Reglamento fue elaborado, emitirá su dictamen sobre los posibles efectos en la salud de las personas, pero sólo a petición de la Comisión, que tendrá esta opinión en cuenta (art. 10.3). La solicitud finalizará con un acto

29 «Si las medidas previstas no son conformes al dictamen del Comité o en caso de ausencia de dictamen, la Comisión presentará sin demora al Consejo una propuesta relativa a las medidas que vayan a adoptarse e informará al Parlamento Europeo", Decisión del Consejo de 28 de junio de 1999 por la que se establecen los procedimientos para el ejercicio de las competencias de ejecución atribuidas a la Comisión, art. 5. 
de ejecución (art. 10.5). La Comisión presentará al Comité Permanente de Vegetales, Animales, Alimentos y Piensos el proyecto de acto de ejecución (art. 12), que se adoptará si el Comité emite un dictamen favorable (Reglamento 182/2011, art. 5) ${ }^{30}$.

Además de los requisitos generales (pruebas científicas que demuestren que el nuevo alimento no plantea un riesgo para la salud, y, «en su caso, el método o los métodos de análisis» ${ }^{31}$ ), en el caso de los nanomateriales hay requisitos más específicos: si hay métodos de ensayo que se apliquen a los nanomateriales artificiales, debe facilitarse «una explicación de su idoneidad científica para los nanomateriales» (art.10.4), por lo que respecto a los métodos utilizados los requisitos son más estrictos en el caso de los nanomateriales artificiales.

En definitiva, el procedimiento se ha simplificado, ya que anteriormente el Estado miembro encargaba una evaluación inicial si bien, si no se necesitaba una evaluación complementaria o se formulaba una objeción de conformidad, el mismo Estado miembro tomaba la decisión. La evaluación por el Estado miembro se ha eliminado y también su facultad para decidir si el alimento se comercializa, ya que el procedimiento se ha centralizado. Por otra parte, esto implica a su vez una mayor coherencia en la autorización de los nuevos alimentos. En la regulación actual, la EFSA realiza una evaluación, sólo si la solicita la Comisión. Esto implica que se pierde capacidad de elección respecto a los organismos de evaluación. En ambos casos se prevé la intervención de un Comité, pero en el Reglamento de 1997 esta intervención sólo tenía lugar si se necesitaba una evaluación complementaria o se formulaba una objeción de conformidad, mientras que en el Reglamento de 2015 este Comité interviene en todos los casos, ya que la Comisión debe presentarle el proyecto de acto de ejecución. HYDE, HARTLEY y MILLAR destacan que «el espacio de discusión que permitía [el reglamento] 258/97 tenía el potencial de mejorar, de manera coope-

30 Si no hay dictamen o el dictamen es desfavorable, la Comisión podrá adoptar el proyecto de acto de ejecución «cuando deba adoptarse sin demora con el fin de evitar perturbaciones significativas en los mercados en el sector de la agricultura o un riesgo para los intereses financieros de la Unión en el sentido del artículo 325 del TFUE.

En tal caso, la Comisión presentará inmediatamente al comité de apelación el acto de ejecución adoptado. Cuando el comité de apelación emita un dictamen no favorable sobre el acto adoptado, la Comisión revocará ese acto de inmediato. Cuando el comité de apelación emita un dictamen favorable o no emita ningún dictamen, el acto de ejecución seguirá en vigor» ( Reglamento 182/2011, art. 7).

31 Reglamento 2015/2283, art. 10.2.e y f. 
rativa, la capacidad de los negocios y el regulador a través de un diálogo experto» ${ }^{32}$.

Por último, hay que tener en cuenta que son las mismas empresas solicitantes las que comprueban si un alimento cae dentro del ámbito del Reglamento y necesita autorización, de acuerdo con el art. 4 («los explotadores de empresas alimentarias comprobarán si el alimento que quieren comercializar en la Unión entra en el ámbito de aplicación del presente Reglamento»), mientras que en el anterior Reglamento, cuando alimentos de algunas de las categorías de nuevos alimentos eran sustancialmente equivalentes a alimentos o ingredientes alimentarios existentes se notificaba la puesta en el mercado de estos a la Comisión (arts. 3.4 y 5). La necesidad de notificación se ha eliminado, lo que puede, según HYDE, HARTLEY y MILLAR «llevar a que (...) de manera potencial, más alimentos no autorizados entren en el mercado ${ }^{33}$. Como afirman estos autores, «los requisitos de aprobación de equivalencia sustancial proporcionaban un espacio para la consulta y la comunicación antes de la comercialización del alimento» ${ }^{34}$.

\section{CONCLUSIONES}

De acuerdo con el artículo 168.1 del TFUE «al definirse y ejecutarse todas las políticas y acciones de la Unión se garantizará un alto nivel de protección de la salud humana». Este aspecto es especialmente relevante en el ámbito alimentario, ya que la alimentación tiene un impacto importante en la salud. En el contexto de la circulación e introducción de alimentos provenientes de otros países y de las nuevas tecnologías que modifican los alimentos, la regulación alimentaria es fundamental para garantizar que la ciudadanía adquiera alimentos seguros.

En relación con los nuevos alimentos, es necesario comprobar que estos no plantean un riesgo para la salud de las personas antes de comercializarlos, por lo que existe un procedimiento de autoriza-

${ }^{32}$ HYDE, R., HARTLEY, S., MILLAR, K., «European Novel Foods Policy at a critical juncture: drawing lessons for future Novel Food Governance through a retrospective examination of Regulation 258/97», Food and Drug Law Journal, 72 (3), págs. $472-505$, pág. 502 : «The space for discussion allowed by $258 / 97$ had the potential to cooperatively enhance the capacity of the business and the regulator through an expert dialogue».

33 Ibid., pág. 501: «may lead (...) potentially to more unapproved novel foods entering the market and the associated risks to consumer protection».

${ }^{34}$ Ibid. 
ción. Era necesaria una actualización en este campo, ya que el Reglamento de 1997 no incluía algunas de las categorías de alimentos que ahora se han añadido, debido en parte al progreso tecnológico y científico. Es el caso de los alimentos consistentes en nanomateriales artificiales, que no se encontraban en el Reglamento de 1997 y han sido incluidos ahora en el nuevo Reglamento. Esto supone una mayor protección de la salud, ya que, anteriormente, al no estar regulados no debían pasar el procedimiento de autorización.

A pesar de la mejora que supone la actualización de la regulación en este ámbito, deben considerarse algunas cuestiones problemáticas en relación con este Reglamento.

En primer lugar, aunque este incluye una definición de nanomaterial artificial, en otros campos relacionados con los alimentos se mencionan la sustancias en nanoforma y las nanopartículas, pero no se definen. Esto puede producir cierta incertidumbre. En otros ámbitos no alimentarios existen definiciones distintas, como la establecida de manera general en la Recomendación de la Comisión de 2011, que define "nanomaterial», o la establecida en el Reglamento 123/2009 sobre los productos cosméticos. En todo caso, el Reglamento de 2015 asume la necesidad de coherencia respecto a la definición de nanomaterial artificial en el ámbito alimentario. De este modo, establece que el Reglamento 1169/2011 sobre la información alimentaria facilitada al consumidor remita al Reglamento de nuevos alimentos en lo referente a la definición de nanomaterial artificial.

También debe tenerse en cuenta que la definición incluida en el Reglamento de 2015 se adaptará a los progresos técnicos y científicos, lo que, aun siendo necesario, puede significar una mayor inseguridad en este campo.

En segundo lugar, la centralización del procedimiento implica que pueda existir una mayor coherencia en la autorización de nuevos alimentos. No obstante, también significa que se han reducido un poco las posibilidades de evaluación. Por otro lado, aunque haya menos pasos en este proceso, todavía se considera un procedimiento largo.

Por último, ha desaparecido la necesidad de notificar la puesta en mercado de los alimentos sustancialmente equivalentes a los existentes. Los explotadores de empresas alimentarias deben comprobar si el alimento entra en el ámbito de aplicación del Reglamento de nuevos alimentos y no tienen que notificar si ponen en el mercado un alimento sustancialmente equivalente. Esta eliminación de la no- 
tificación puede llevar a que más alimentos no autorizados entren en el mercado.

En definitiva, el Reglamento de nuevos alimentos del año 2015 es una mejora necesaria de la protección de la salud respecto a la situación anterior, ya que introduce nuevas categorías que antes no estaban reguladas. Sin embargo, aspectos como la no homogeneidad de definiciones o ausencia de estas, así como la mayor autonomía en la decisión de lo que es un nuevo alimento de las personas solicitantes y la duración del proceso, podrían mejorarse para una protección más efectiva de la salud. 